\title{
Subglottic stenosis as a complication of Wegener's granulomatosis
}

\author{
*Allen J Blaivas ${ }^{\mathrm{a}}$, Walter Strauss ${ }^{\mathrm{b}, c}$, Michael Yudd ${ }^{\mathrm{b}, \mathrm{d}}$ \\ a Division of Pulmonary, Critical Care, and Sleep Medicine, Department of Veterans Affairs, VA New Jersey Health Care System, 385 \\ Tremont Avenue, East Orange, NJ 07018, USA \\ ${ }^{b}$ Assistant Professor of M edicine, UMDNJ - New Jersey Medical School \\ ' Chief, Division of Pulmonary, Critical Care, and Sleep M edicine, Department of Veterans Affairs, VA New Jersey Health Care System \\ d M edical Director, Dialysis Unit, Division of Nephrology and Hypertension, Department of Veterans Affairs, VA New Jersey Health Care \\ System
}

Received 18th January 2007; accepted 23rd October 2007

\section{Summary}

Wegener's granulomatosis (WG) is a relatively uncommon collagen vascular disease that can lead to both upper and lower airway disease. Subglottic stenosis is one manifestation of the airway disease and can occur even during the quiescent phase of the disease, independent of an active inflammatory response. A high index of suspicion is necessary for this complication in patients with known WG who complain of dyspnoea, and WG should be high on the differential diagnosis in those found to have "idiopathic" subglottic stenosis. We report the case of a patient who presented with this problem, and we review the prevalence, diagnosis, symptoms, and treatment.

(c) 2008 General Practice Airways Group. All rights reserved.

AJ Blaivas et al. Prim Care Resp J 2008; 17(2): 114-116.

doi:10.3132/pcrj.2008.00002

Keyw ords Wegener's granulomatosis, dyspnoea, stridor, subglottic stenosis, diagnosis

\section{Introduction}

Wegener's granulomatosis (WG) is an uncommon necrotising vasculitis that affects the airways, sinuses, and kidneys. We present a patient with WG on immunosuppressive therapy who developed progressive dyspnoea and was found to have an unusual, and often overlooked, airway complication of this disease.

\section{Case report}

A 55 year-old white male with a past medical history of Wegener's granulomatosis was admitted to our hospital with complaints of progressive dyspnoea on exertion, and stridor that occurred only on forceful breathing. His WG had been diagnosed approximately nine months previously, and his presentation at diagnosis was significant for recurrent rhinosinusitis, arthralgias, active urine sediment, and ulcerating tracheitis. Kidney biopsy revealed crescenteric glomerulonephritis and C-ANCA on diagnosis was 1:640. He had been started on treatment with cyclophosphamide and prednisone, and was still on this treatment when admitted nine months later.

On admission, the patient denied any arthralgia or active otorhinolaryngological manifestations of the disease. Urine sediment was bland, and C-ANCA was only 1:80. However, in view of the previously noted tracheitis caused by WG at diagnosis, upper airway obstruction was suspected. Variable extrathoracic obstruction was discovered on flow volume loop (see Figure 1), and CT scan revealed critical narrowing in the subglottic region extending for almost $15 \mathrm{~mm}$, with the thinnest diameter being approximately $6 \times 5 \mathrm{~mm}$ (Figure 2). Bronchoscopy confirmed the critical stenosis immediately below the vocal cords. The stenotic area did not appear inflamed on bronchoscopic examination.

The patient underwent tracheostomy to secure the airway and he has been undergoing combination treatment with intralesional glucocorticoid therapy and serial passage of blunt dilators to enlarge the stenotic airway. 


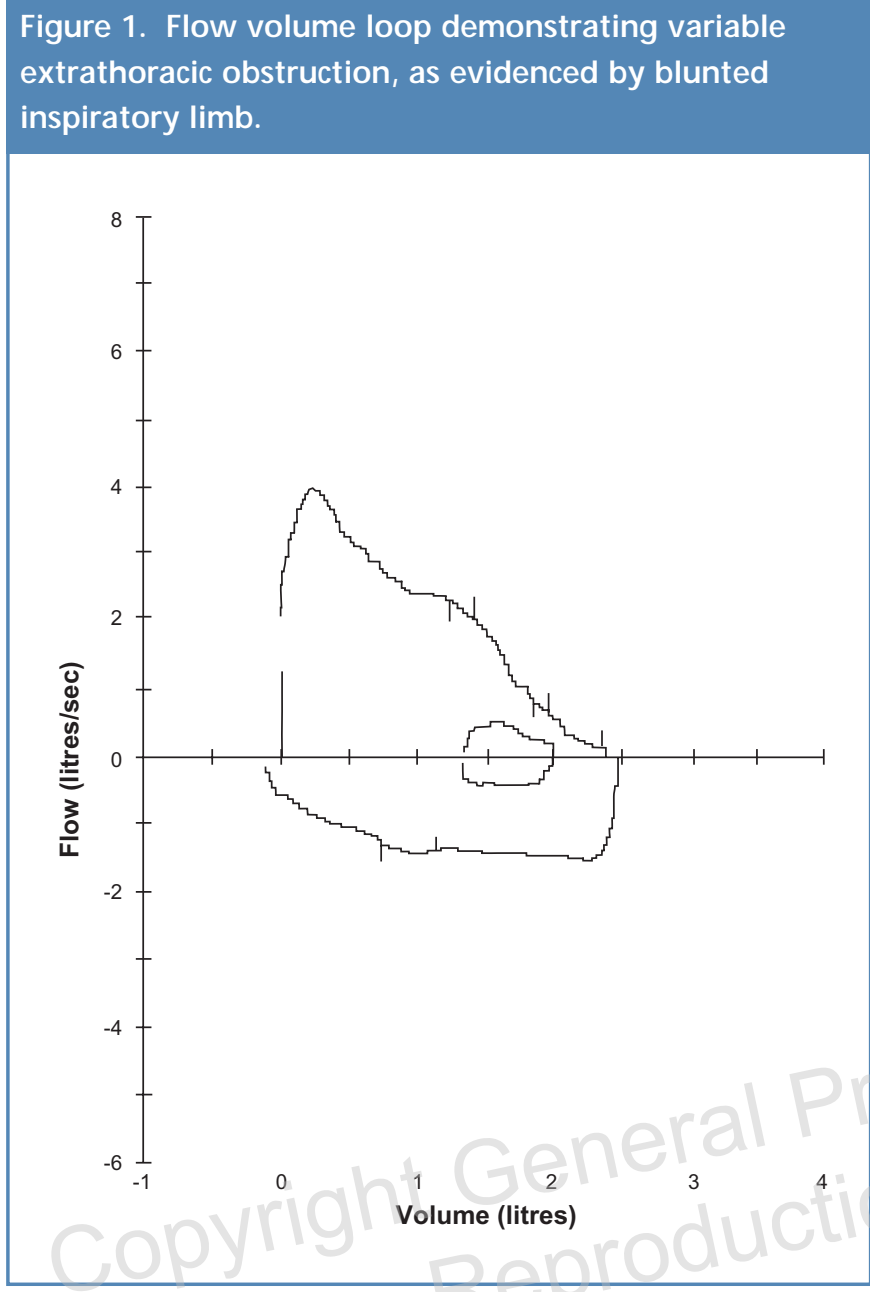

\section{Discussion}

Wegener's granulomatosis most commonly affects the upper and lower respiratory tract, although any organ system may be involved. Airway and pulmonary complications of WG include subglottic stenosis, tracheal and endobronchial inflammation and stenoses, granulomatous pulmonary nodules, and alveolar and cavitary infiltrates. ${ }^{1} \mathrm{As}$ was the case in our patient, the upper respiratory tract disease commonly presents as rhinosinusitis. Also noted at initial diagnosis was a lower airway manifestation of WG, ulcerating tracheitis.

Subglottic stenosis (SGS) occurs in approximately $16-23 \%$ of patients with WG sometime during the course of disease and results from circumferential inflammation, edema, and fibrosis that typically extends for 3 to $4 \mathrm{~cm}$ below the vocal cords. The subglottic region of the trachea is particularly susceptible to symptomatic narrowing due to the complete ring that comprises the cricoid cartilage - in contrast to the lower trachea which has a soft fibromembranous posterior wall. The normal subglottic area in the adult patient has an internal diameter of $23 \mathrm{~mm}$ laterally and an anteroposterior diameter of $18 \mathrm{~mm}$.

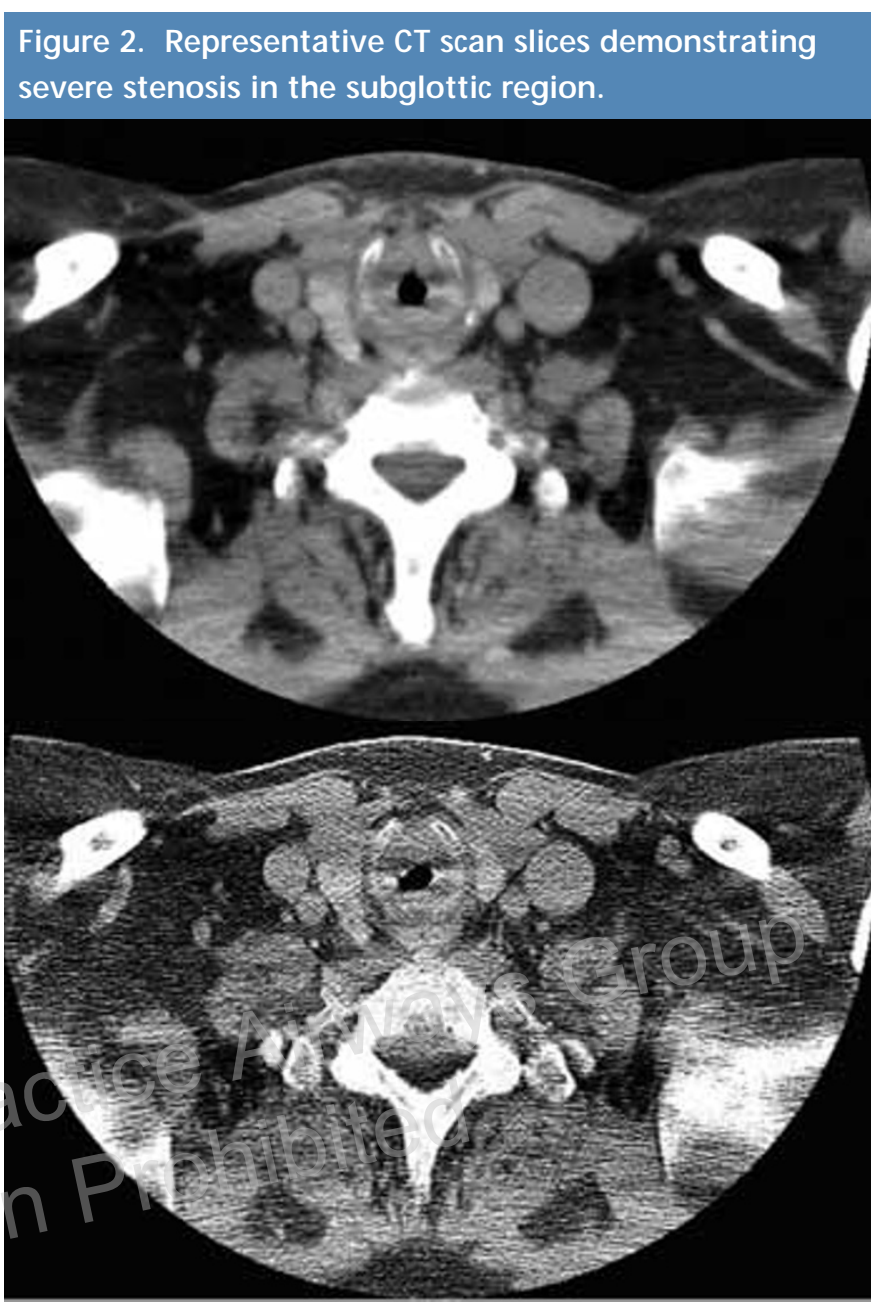

Dyspnoea on exertion and stridor are the most common presenting complaints $-81.5 \%$ and $29.6 \%$, respectively. However, depending on the degree of narrowing, patients may be asymptomatic. ${ }^{2}$ In our case the relationship to WG was obvious, but the differential diagnosis of SGS can include trauma ( $90 \%$ of which is due to endotracheal intubation), infections such as tuberculosis, diphtheria, or syphilis, inflammation due to sarcoidosis or relapsing polychondritis, chemical irritation, and obstruction by a foreign body. ${ }^{3}$ SGS is often seen on the flow-volume loop, with the characteristic finding of fixed airway obstruction. ${ }^{1}$ Our patient had the less common finding of variable exthoracic obstruction demonstrated on the inspiratory curve. When noted, bronchoscopy and/or CT scan of the neck, particularly with virtual bronchoscopy technology, may be useful in confirming the diagnosis. ${ }^{4}$

As seen in our patient, SGS - which is not necessarily associated with acute inflammation - may occur during the quiescent phase of the illness, and is caused by mature subglottic scarring. ${ }^{4}$ In our patient all other manifestations of the disease were relatively controlled when the patient began 


\section{Learning Points}

1. Subglottic stenosis is a common cause of dyspnoea in patients with Wegener's Granulomatosis and occurs even when systemic inflammation is well controlled.

2. Diagnosis can be obtained with CT scan of the neck, preferably with virtual bronchoscopy, flow-volume loop, and endoscopic visualisation.

3. Intratracheal dilatation, often with intralesional glucocorticoids, is usually necessary when a symptomatic narrowing is present.

developing the complaint of dyspnoea that was subsequently revealed to be caused by SGS.

Although some patients respond to systemic immunosuppressive therapy, manual dilation with intralesional corticosteroids, often preceded by tracheostomy, is required in most symptomatic patients. Laser resection is not beneficial, since it usually leads to extensive scarring and stenosis. $^{5}$

\section{Conclusion}

Though uncommon, clinicians should strongly consider a diagnosis of SGS in any patient with a known history of WG who presents with dyspnoea. Conversely, WG must be ruled out in any patient that is found to have SGS.

\section{Conflict of interest declaration}

None declared.

\section{References}

1. Arcasoy SM, Kreit JW. Recurrent sinusitis, arthralgias, and progressive dyspnea in a 26 year old woman. Chest 1999;115(6):1731-4.

2. Gluth MB, Shinners PA, Kasperbauer JL. Subglottic stenosis associated with Wegener's granulomatosis. Laryngoscope 2003;113:1304-07.

3. Langford CA, Sneller MC, Hallahan CW, et al. Clinical features and therapeutic management of subglottic stenosis in patients with Wegener's granulomatosis. Arthritis Rheum 1996;39(10):1754-60.

4. Summers RM, Aggarwal NR, Sneller MC, Cowan MJ, Wood BJ, Langford CA, Shelhamer JH. CT virtual bronchoscopy of the central airways in patients with Wegener's granulomatosis. Chest 2002;121(1):242-50.

5. Lebovics RS, Hoffman GS, Leavitt RY, et al. The management of subglottic stenosis in patients with Wegener's granulomatosis. Laryngyscope 1992; 102:1341-5.

\section{Available online at http://ww w.thepcrj.org}

